\title{
JUVENTUD Y MOVIMIENTOS SOCIALES: REFLEXIONES SOBRE LA GENERACIÓN GLOCAL LATINOAMERICANA
}

\author{
Youth and Social Movements: \\ Reflections on the Latin American Glocal Generation
}

Francisco Ramírez Varela

framirezv@udla.cl

https://orcid.org/0000-0002-7571-9728

Facultad de Ciencias Sociales Universidad de las Américas

Chile

\section{Resumen}

Palabras clave

juventud movimiento social participación juvenil generación

Keywords

youth

social movement youth participation generation

La juventud ha tendido ha ser caracterizada mediante aspectos que se atribuyen a una generación producto de los procesos culturales e históricos que vive un grupo etario determinado pero sin atender a sus elementos distintivos. En América Latina, muchas de estas distinciones fueron cruzadas por elementos sociohistóricos, entre los que destaca la influencia de los movimientos sociales. En este artículo, el autor propone que la generación actual no es una excepción: en la segunda década del siglo XXI, los/as jóvenes se caracterizan por incorporar en sus movimientos sociales una ciudadanía y una cultura de lo global atravesadas por una perspectiva local, con énfasis en los elementos de socialización y de participación virtual que deben considerase para entender esta Generación Glocal.

\section{Abstract}

Youth is characterized many times within generalized aspects of a generation, understanding this as the cultural and historical processes that a specific age group lives, without keeping the distinctive elements of them. In Latin America, many of these distinctions were crossed by socio-historical elements, among which the influence of social movements stands out.In this article, the author proposes that the current generation is no exception: young people in the second decade of the XXI century, are characterized by the incorporation into their social movements of a citizenship and culture of the global crossed with the local perspective, with emphasis on the elements of socialization and virtual participation that must be considerer to understand this Glocal Generation. 


\title{
JUVENTUD Y MOVIMIENTOS SOCIALES: REFLEXIONES SOBRE LA GENERACIÓN GLOCAL LATINOAMERICANA
}

\author{
Por Francisco Ramírez Varela
}

\section{Consideraciones primeras sobre generación}

La juventud ha sido identificada a partir de su referente demográfico y/o legal bajo un criterio de universal, dentro de determinados rangos de edad. Pero ser mayores de dieciocho años no es la única característica que determina la distinción como población juvenil, sino que existen otras distinciones; entre ellas, el pertenecer a una generación. En este sentido, entendemos que, si bien cuando se habla de generación se hace referencia a un grupo de edad, esta va más allá de dicha característica e incluye la época en que cada persona se socializa. Es decir, se remite a la edad, pero atravesada por la cultura y por la historia (Margulis \& Urresti, 1998).

De lo anterior, podemos inferir que las diferencias generacionales están ligadas a las diferencias, pero también a las herencias de cada generación. Como señala Mario Margulis (1998): «Existen los relatos, la memoria social, la experiencia trasmitida, pero, sin embargo, cada generación se presenta nueva al campo de lo vivido, poseedora de sus propios impulsos, de su energía, de su voluntad de orientar sus fuerzas» (p.3). Estas experiencias y la memoria social refieren a las demandas históricas, que han sido trasmitidas de generación en generación pero con diferencias en el plano de la memoria.

Utilizar el concepto de generación también ha servido para construir sobre la gente joven de determinada época algunos estereotipos (Alpízar \& Bernal, 2003) mediante los cuales se generalizan las características de la juventud acorde a su contexto y a sus características diferenciadores, las que se construyen a partir de su inserción social, caracterizada por sus formas de participación y de socialización. En este sentido, hay que destacar que las generaciones no son estructuras cerradas inalterables, sino referentes simbólicos que apoyan a identificar a los actores socializados en unas mismas coordenadas temporales (Feixa, 2000). 
En la historia del siglo $\mathrm{XX}$, y en lo que hemos avanzado del siglo $\mathrm{XXI}$, puede observarse la sucesión de diferentes generaciones de jóvenes que irrumpen en la escena pública para ser protagonistas en la reforma, la revolución, la guerra, la paz, el rock, el amor, las drogas, la globalización o la antiglobalización (Feixa, 2006). Sin embargo, para el análisis de las generaciones que pretendemos abordar, es importante considerar las variables que influyen en su interpretación.

La primera consideración se relaciona con el hecho de que los diferentes estudios o ensayos son realizados con la intención de clasificar y de describir características generacionales, tarea que, en su gran mayoría, es efectuada desde una visión adultocéntrica que refleja la visión de pasividad y de inactividad de la juventud. En general, esto sucede debido a que la comparación se realiza con las generaciones propias; es decir, con la generación de quien investiga.

La segunda consideración a tomar en cuenta es que las visiones generacionales tienden a una descripción sustentada en criterios eurocentristas, en función de características históricas influyentes y de rasgos de cultura predominantes, muchas veces, alejados de la realidad del caso a analizar, como sucede con América Latina. Un eurocentrismo entendido no solo desde la concepción occidentalista de las generaciones, sino también desde su construcción historiográfica, más asociada a los fenómenos europeos. Así, por ejemplo, advertimos la clasificación de una generación «Babyboomers» (Feixa, 2006), como aquella pos segunda guerra mundial que fue artífice de la reconstrucción del continente. Un eurocentrismo también universalista (Wallerstein, 1999) en cuanto a la construcción de generaciones con características globales, muchas veces alejadas de la realidad y del contexto latinoamericano.

Una tercera consideración remite a la utilización y a la clasificación de las generaciones a partir de un marketing consumista y de la estigmatización mediática. Tal es el caso de las características atribuidas a generaciones como las llamadas «Generación X» o «Millenians» (Ferrer, 2010), para las cuales se hace énfasis en su cultura consumista, que cruza sus hábitos y su socialización. Estas clasificaciones, que atribuyen a todas las personas jóvenes las mismas características, se han vuelto mediáticas, generándose desde ahí todo tipo de reportajes y/o telereportajes basados en estos conceptos generacionales y en los términos de referencia, sea como Generación X, Generación Millennials o Centennials, a partir de conceptualizaciones alejadas de una realidad generacional. 
Una cuarta consideración que debemos atender es que las generaciones no se limitan a los cortes temporales y/o a las edades de los actores sino a procesos históricos que, si bien están marcados dentro de espacios temporales en los que confluyen los mismos elementos dialécticos, no siempre pueden limitarse a un cambio de año o de década. El circunscribir una generación a procesos temporales será un ejercicio academicista, pero no una ley categórica.

La última consideración es que nos encontramos ante una sociedad globalizada y cada vez más compleja: así como no podemos hablar de una sola juventud, sino de juventudes (Duarte Quapper, 2000), tampoco podemos hablar unívocamente de una sola generación, sino de generaciones, o, como menciona Beck (2008), de varias "constelaciones generacionales transnacionales»; que presentan elementos diferenciadores según las características de cada una de las regiones y territorios, como es el caso de América Latina. Frente a esta última, sin embargo, y como señala Carlos Feixa (2006), está pendiente la revisión y la construcción de los elementos alternativos que tengan en cuenta a las «otras juventudes» - no occidentales, rurales, trabajadoras, femeninas-. Por lo tanto, es necesario continuar construyendo esbozos que nos permitan diferenciar los procesos generacionales en América Latina.

\section{Las generaciones en América Latina}

En América Latina, el auge de los estudios sobre el fenómeno juvenil se produce a finales de los años cincuenta y durante los sesenta, en cuanto desde las ciencias sociales «tuvieron que hacer de la realidad juvenil un fenómeno estudiable» (Feixa, 2006, p. 15). De allí que no sea una cuestión menor la necesidad de realizar, en función de los antecedentes de estas miradas, una revisión de las características fundamentales de las generaciones latinoamericanas, tomando como base los procesos históricos sociales propios de la región. De la consideración de los fenómenos generacionales en América Latina, específicamente realizada desde una perspectiva que atienda su influencia en los movimientos sociales, se desprende que las diferentes generaciones poseen elementos distintivos, que no son unívocos ni determinantes. 
Si hacemos un escrutinio de las últimas décadas como etapas generacionales precedentes a la actual, podemos observar que en América Latina los/as jóvenes de la década del sesenta representaron el ímpetu rebelde, reflejado en los primeros levantamientos estudiantiles, en el surgimiento de los movimientos revolucionarios, de la adaptación del marxismo latinoamericanizado; en fin, de la creación de los movimientos y frentes nacionalistas.

La década de los sesenta se presenta marcada por la escena global, por el reflejo de relaciones políticas de más de medio siglo de historia: el fin de la II Guerra Mundial y una nueva configuración geopolítica internacional, en la que se distinguen la hegemonía del mundo capitalista y la hegemonía del bloque soviético en el mundo socialista. Basta como ejemplo la mención del muro que mantenía un mundo bipolar, separado en dos bloques, no solo influyentes en lo político, sino con notorias diferencias en lo ideológico, lo valórico y lo teórico. En América Latina, esto se refleja en la Revolución Cubana de 1959, la cual dio pie a la organización y a la consolidación de nuevos partidos de izquierda y de movimientos sociales y armados, muchos de los cuales nacieron en el seno mismo de las universidades y de los/as jóvenes. Entre otros, se puede advertir el surgimiento de importantes movimientos culturales, feministas y raciales, dentro de los cuales no hay que descartar a los movimientos estudiantiles.

Se gesta una reorganización crítica y revolucionaria de los estudiantes universitarios, que se destaca como un referente de la distinción generacional de un movimiento con tintes netamente juveniles. La ola expansiva de los hechos acontecidos en Francia en la primavera de 1968 (conocidos como Mayo del 68 o Mayo Francés) tiene un gran eco dentro del ámbito de los movimientos estudiantiles universitarios en América Latina y encuentra como hecho más significado la matanza de Tlatelolco en Ciudad de México, que marcó a los movimientos estudiantiles reformistas latinoamericanos. Se generan, entonces, los elementos distintivos de lo que se podría llamar una «Generación revolucionaria», no por ser la única, sino por proporcionar los elementos centrales que originan el accionar de esta generación y que la marcarán posteriormente. Se trata, pues, de una generación que busca cambios estructurales, atravesados por fuertes señales ideológicas que se generalizan en la América Latina. 
Los/as jóvenes de la década del setenta, en tanto, no dejaban de inspirarse y de proclamar las ideas revolucionarias, pero en otros contextos, puesto que, en su mayoría, vivieron en el marco de gobiernos represores que determinaron que se tuviera que pasar por procesos de reafirmación de ideologías y de acumulación de fuerzas. Esto se produce, ya que la gran mayoría de países del continente, sea en forma esporádica o permanente, vivió periodos dictatoriales, marcados por los golpes militares y por la represión violenta de las ideologías, fundamentalmente de los movimientos sociales de la generación anterior. La permanente violación de los derechos humanos se ve cruentamente expresada desde los sucesivos gobiernos militares de Guatemala, que durarían hasta mediados de los años ochenta y que implementarían una guerra civil antisubversiva que arrasaría indiscriminadamente la selva del Peten, hasta las dictaduras del Conosur, asociadas con la denominada «Operación Cóndor», que instauró el terrorismo de Estado, asesinando y desapareciendo a los líderes opositores, principalmente vinculados a organizaciones de izquierda.

No hay necesidad de recalcar, pero no es posible dejar de mencionar que, como la gran mayoría de estos/as líderes, los/as dirigentes y los/as ciudadanos/as que fueron violentados/as en sus derechos más básicos fueron en su gran mayoría jóvenes. Esta situación provocó que todo tipo de organización y de movimiento social asociado a los/as jóvenes fuera visto como una amenaza y sufriera los embates de la represión. Del mismo modo, no se debe desconocer que existieron muchos movimientos juveniles asociados a los regímenes dictatoriales y militares, que fueron fuertemente criticados y censurados por gran parte de la opinión pública. Este contexto refirma la descripción de estos/as jóvenes como la «Generación de las catacumbas", en alusión a los movimientos religiosos que durante el imperio romano se ocultaban dentro de las catacumbas para realizar sus actividades sin ser reprimidos.

El joven de la década de los ochenta, en tanto, representa la represión a la vez que la lucha contra las dictaduras en la región y la reconstrucción de la democracia. Representa el resurgimiento de los movimientos juveniles, aparentemente acallados en la generación anterior, pero esta vez en un contexto que se abre a la crítica de los regímenes antidemocráticos, lo que da pie a la posibilidad de manifestar la necesidad de procesos democráticos. La crítica estructural a los sistemas políticos fue conjugadora de esta generación, pero inserta en movimientos sociales y políticos 
mayores que, específicamente en el caso chileno, clamaron por el cambio, por el retorno a la democracia. En América Latina, estos procesos se manifestaron de diferentes formas, sea por el retorno a la democracia o por su fortalecimiento. Es el espíritu de cambio que distinguió a los/as jóvenes de los ochenta lo que permite caracterizarlos como una «Generación del cambio».

En la década de los noventa, la caída de modelos, de ideales, o su no resultado -metafóricamente representado por la caída del muro de Berlín-, determinaron que la juventud pareciera dormida, decepcionada, pero que fuera, a la vez, reflexiva hacia los nuevos sucesos. Los/as jóvenes de esta generación se ubicaron a la sombra de la generación anterior que, de alguna manera, promovió los cambios sociales y estructurales en gran parte de las sociedades latinoamericanas. De allí que, comparativamente, esta generación sea asociado con el estereotipo del letargo. En Chile, por ejemplo, durante la década del noventa se popularizó la frase «no estoy ni ahí», utilizada con frecuencia por un joven deportista que mostraba su desenfado y la poca importancia que le otorgaba a lo que dijeran los medios; misma frase que se acuñó como descriptora de una generación de jóvenes en cuanto a la neutralidad y el desinterés frente a la participación política y al involucramiento en el desarrollo de políticas sectoriales.

Esta suerte de «indiferencia», que coloca a los individuos entre el narcisismo y el desierto posmoderno que enfrentan, también representa una indiferencia a los referentes ideológicos de los modelos políticos en declive durante los años noventa (Lipovetsky, [1986] 2000). Sin embargo, este estigma asumido por los/as propios/as jóvenes haría referencia a su alejamiento de la clase política y de las políticas públicas, así como de las formas de participación convencional y hegemónica. Los/as jóvenes se volcarán a sus propios procesos, a la búsqueda de su propio «ahí»; es decir, a la revisión de su posición dentro del entramado social, realizando un «retorno a lo sagrado» (Lipovetsky, [1986] 2000) y redefiniéndose, así, como una «Generación reflexiva».

La generación de los/as jóvenes que da inicio al siglo XXI puede definirse como la «Generación cero», no solo porque comienza con el nuevo siglo sino porque se inicia, también, desde nuevos paradigmas y formas de socializar la juventud. Empieza a verse con mayor fuerza la influencia de los fenómenos globalizantes, en especial por el caracter transversal de Internet, que amplía el espectro comunicativo y, sobretodo, informacional de los/as jóvenes, cuyas miradas y opciones se globalizan. 


\section{La Generación Glocal}

El transcurso de este siglo no solo marca profundamente la construcción de lo juvenil desde la propia generación, sino que nos enfrenta a jóvenes que adaptan y que adoptan elementos de la globalización a sus propias realidades locales. Los/as jóvenes se movilizan desde una perspectiva global, pero con sus intereses locales bien marcados. Ello se refleja en los movimientos juveniles de la primera etapa del siglo XXI, puesto que si bien las transformaciones sociales se manifiestan en espacios localizados han conseguido trascender las fronteras locales y nacionales (Feixa \& Nilan, 2009). Entre la desconfianza y el desencanto, miles de jóvenes eligen otras formas de participación, más puntual, específica y local (Croce, 2012). Si bien es cierto que adquieren elementos culturales y comunicacionales vertidos por la globalización, los manifiestan a su manera en los espacios locales. Ello nos ubica frente a la construcción de una nueva generación, la «Generación glocal».

Si hablamos de la inserción de los/as jóvenes como una generación glocal, debemos partir de adentrarnos en la búsqueda del contenido conceptual de la globalización, como una palabra que se utiliza en todo tipo de contextos y que en algunos casos es usada de tal modo que pareciera poder atribuirse a este fenómeno todo lo que ocurre en la sociedad y en la economía, cuando en otros contextos se reduce simplemente a un aspecto o una dimensión. Wallerstein (1999), hace hincapié en que la globalización es «un slogan», una etiqueta mediante la cual se puede clasificar o desclasificar los productos, los fenómenos y/o las sociedades actuales.

La primera imagen con la que solemos asociar la conceptualización de globalización es la economía, pero este aspecto, aunque es el más fuerte e injusto en nuestras economías nacionales y locales, no es el único. A la globalización, más que seguir intentando conceptualizarla, hay que revisarla desde las tres dimensiones o aspectos fundamentales que la integran. El primero, sin duda, tiene relación con el ámbito económico, pero también están las dimensiones políticas y socioculturales. Todo ello con un eje transversal de vital importancia: las Tecnologías de la Información y Comunicación (TIC) y su incesante crecimiento, proceso en el que la globalización ha intervenido al abrir, facilitar y dinamizar el desarrollo de los adelantos tecnológicos que permiten, a la vez, una mayor fluidez en los diversos aspectos de la información, contribuyen al crecimiento de las diferentes relaciones y convierten a los/as jóvenes en sus principales usuarios/as, en los/as protagonistas de la globalización (Ramirez, 2014). 
La globalización es un proceso histórico debido a su capacidad para generar su propia historia. Aunque no hay una delimitación precisa de su influencia, sí existe un reflejo claro que tiene su principal connotación en el ámbito local, donde su presencia genera secuelas, beneficios y consecuencias; de allí que lo que es un fenómeno global se convierta en una realidad local. Surge, de este modo, un elemento esencial en la comprensión de la globalización y de las nuevas formas de interacción entre los actores globales y locales. Al respecto, Roland Robertson y Kathleen White (2004) acuñaron el término glocalización, que se asocia a la globalización de los fenómenos pero, al mismo tiempo, a la localización de sus relaciones económicas y políticas. El neologismo alude, así, a las relaciones que se vienen entretejiendo entre las esferas de lo global y lo local.

En este entramado de lo glocal aparece representada la generación de jóvenes del último decenio; asumiendo que la globalización es un proceso que persiste en la vida diaria y en la concepción de la cotidianeidad de nuestras sociedades, donde más que un concepto y un proceso es una realidad que gravita sobre ella y que convierte a la glocalización en una realidad cotidiana. Una realidad que se ve reflejada en las prácticas de las personas, con mayor énfasis en la población joven, en sus expresiones culturales, pero también en sus formas de asociatividad, lo que genera cambios en la producción de sus demandas sociales y de sus formas participativas.

Es indispensable tener en cuenta la incorporación de las TIC en la construcción de una nueva generación glocal y en sus mecanismos de participación. Apoyada en las innovaciones tecnológicas, la participación juvenil actual adquiere una fuerte capacidad articuladora a nivel nacional, regional y global. Los/as jóvenes que están comprometidos con una causa local conocen bastante de cerca lo que sucede en otros lugares y, no pocas veces, tienen relaciones directas con otros/as jóvenes que luchan por causas similares en otros puntos de América Latina y del planeta (Croce, 2012). En este sentido, la presente generación ha encontrado nuevos modos de movilizarse y se sabe en los medios de comunicación; existe, por así decirlo, una «consciencia mediática» arraigada en ellos/as (Cuadra, 2012).

Los nuevos paradigmas nos posicionan ante una generación que es protagonista de su propia construcción, desde los procesos de socialización y de participación en movimientos sociales. Los/as jóvenes comienzan a ser constructores/as de nuevas realidades, al tiempo que surgen nuevos factores movilizadores que se enmarcan en nuevos contextos, claramente influenciados por fenómenos globalizantes. 
Los nuevos movimientos sociales no se plantean un ataque revolucionario contra el sistema sino que más bien reclaman a los gobiernos democráticos que abran la vida política al conjunto de intereses relacionados con los ciudadanos (Feixa \& Nilan, 2009). A diferencia de lo observado en las generaciones anteriores, los/as jóvenes militantes de las primeras décadas del siglo XXI no se plantean construir «movimientos juveniles» u «organizaciones juveniles». Su perspectiva más bien es intergeneracional, enfocada en espacios o en situaciones locales, salvo casos muy particulares, como el de los movimientos estudiantiles (Croce, 2012).

Es de esperarse entonces, la construcción sobre las juventudes de nuevas miradas desde lo glocal, las cuales deberán atender a aquellos elementos que se encuentran transversalmente dentro del concepto de generación: la cultura glocal, la ciudadanía glocal y lo virtual.

Es así que las culturas juveniles se ven afectas a los procesos globales expandiendo multiidentidades culturales que adoptan prácticas socioculturales de diversas latitudes, adaptadas a las culturas y a las realidades locales. Muchas de estas son representadas por las agrupaciones entendidas como "tribus urbanas», que más allá del concepto primario elaborado por Michel Maffesoli (1998), son grupos con ciertas características y significados comunes que reflejan esa adaptación cultural. A manera de ejemplo, podemos considerar a los punks que adaptan las características indígenas a su estética o a los seguidores de culturas asiáticas que realizan "cosplay» (caracterizaciones de animaciones japonesas) en torno a sus propios contextos.

Esta cultura no solo es glocal en su actitud y en sus prácticas sociales, también es transversal en sus formas de involucramiento social, aspecto que se refleja en los movimientos sociales. Se produce, de este modo, un cambio en las formas de participación de los/as jóvenes por medio de la inserción de elementos culturales característicos de la generación, que trascienden en lo informal y que se insertan y se adoptan en las estructuras de la participación organizada.

De este modo, los movimientos sociales se ven impregnados de nuevas formas de participación, que resultan innovadoras y que rompen el esquema tradicional. Vemos los movimientos sociales y sus manifestaciones diferentes: flashmobs (acciones estéticas instantáneas y organizadas) convocados durante el movimiento, con acento en los elementos culturales de los/as jóvenes, que incorporan en su 
manifestación música, baile $\mathrm{y} / \mathrm{o}$ caracterizaciones que rompen los esquemas tradicionales de una marcha de protesta -a veces, incluso, con toques "carnavalescos»-, pero que desde la mirada de la propia generación tienen un sentido y una representatividad de su propia cultura.

Como segunda característica se encuentra la ciudadanía glocal, entendida desde la concepción básica como todos aquellos que son sujetos de derechos y deberes. Los movimientos glocales juveniles son parte de la expresión de una nueva ciudadanía, empoderada de su espacio, que participa en el entorno local pero que se manifiesta globalmente. Se trata, pues, de una ciudadanía con derechos que son globales, pero con un fuerte compromiso para con sus deberes locales. Existe, por tanto, una mayor información sobre derechos, así como sobre sistemas y políticas sociales globales, de la misma forma que se produce una mayor adhesión a reivindicaciones sostenidas por diferentes movimientos en torno al orbe. Tal es el caso de las manifestaciones de indignados o de los movimientos ecologistas que si bien son globales toman para sus demandas elementos de importancia local.

Un ejemplo de cómo sus deberes se enfocan sobre todo en lo local es la medición de las instancias de participación. Por un lado, se evidencia una menor participación en el ámbito de lo político entendido como la política institucionalizada, lo que alcanza un mayor reflejo en los procesos eleccionarios. Por el otro, se observa en el incremento de la participación en ámbitos locales, como los ámbitos de estudio y/o la comunidad, donde existen intereses más inmediatos, demandas específicas y, muchas veces, la construcción de respuestas y de soluciones colectivas.

De manera transversal a la cultura y a la ciudadanía glocal, aparece una tercera característica ya mencionada: lo virtual. El desarrollo de los espacios virtuales, por medio de las nuevas tecnologías, se identifica con las nuevas formas de comunicación y de información, que acompañan los cambios paradigmáticos en el ámbito de lo sociocultural. Este desarrollo de las TIC tiene su expresión a través de Internet, en tanto canal de la globalización de las comunicaciones. Frente a esto, si bien es cierto que el acceso cada vez más global a los medios de comunicación y a la modernización no impide que esta continúe siendo exclusiva y elitista, no debemos hacer ojos ciegos el aumento de la conectividad en América Latina, donde, proporcionalmente, la gran mayoría de las conexiones corresponde a la población joven. 
La brecha digital que se ha producido por la penetración de las nuevas tecnologías de comunicación se ha ido transformando en una brecha generacional. Los/as jóvenes constituyen una generación «nativa digital» (Piscitelli, 2005), que le da una nueva utilización a las TIC y las incorpora en sus procesos de socialización y de participación. En este sentido, Internet no solo es considerada como un complejo conjunto de tecnologías de comunicación sino también como una tecnología que permite nuevas formas de representación y que, por lo tanto, contribuye a la reconfiguración del sujeto contemporáneo en tanto agente de prácticas virtuales que se articulan con su realidad sociocultural.

El aumento de la información es proporcional al crecimiento de la interconectividad en el ciberespacio, donde no solo se da el proceso de la inteligencia colectiva por medio del intercambio de información, sino que en parte es su acceso lo que permite incorporar nuevos elementos a su cotidianidad. De este modo, la utilización de redes sociales virtuales es un soporte de transformación de los tipos de enlaces, que facilitan el crecimiento de la red de contactos y que crean procesos diferentes en la cercanía y en la concreción de enlaces fuertes.

Si bien es cierto que Internet y las redes sociales no son generadoras de transformaciones sociales, sino facilitadoras en el proceso de canalización de la comunicación y la información, a las que aceleran y diversifican, el papel que juegan las redes sociales en Internet es el punto de partida para explicar buena parte de las dinámicas sociales en las que los/as jóvenes se encuentran inmersos.

Los movimientos sociales de los últimos años ponen de manifiesto que para esta generación el uso de redes sociales ha sido uno de los elementos fundamentales tanto para la promoción y la coordinación de las acciones organizadas, como para su difusión y socialización; lo que refleja la característica de participación informal que distingue a esta generación como producto de la socialización virtual. De esta forma, Internet se convirtió en uno de los aliados más importantes de los movimientos sociales: «Del megáfono a la Internet, en cada momento histórico la juventud es el segmento de la sociedad que busca utilizar de forma más creativa las modernas herramientas comunicacionales para expresarse» (Mielli, 2012, p. 13). 


\section{Elementos para la reflexión}

Los elementos descritos que distinguen esta generación pueden ser entendidos desde la manifestación de los procesos de glocalización en los que están insertos; características que se encuentran presentes en su actuar cotidiano pero, sobre todo, como un elemento diferenciador en sus manifestaciones asociativas, en función de construir movimientos sociales. Es por ello que hacemos énfasis en el hecho de que los movimientos juveniles que en su gran mayoría se inician en contextos locales luego tendrán una repercusión macro y global.

En la actualidad, la diversidad de movimientos sociales confluye en diversos aspectos y tiene muchos de los aspectos descritos. Esto puede observarse en los movimientos indigenistas a lo largo del continente, como sucede con el reconocimiento de la lucha del pueblo mapuche a nivel global y, como no mencionar, con los zapatistas en Chiapas, que marcan una pauta innegable en cuanto a movimientos glocales.

Lo mismo sucede con las agrupaciones medioambientalistas, que si bien parten de una realidad específica se globalizan en sus alcances, como sucede con el movimiento «Patagonia sin represas» o con la defensa de la Amazonía; pero, aun así, es la juventud que mejor refleja estos nuevos movimientos, dadas las características glocales mencionadas. $Y$ es que los/as jóvenes no solo conocen el manejo de las herramientas y las tecnologías, sino que también saben aprovecharlas, lo que les permite masificar sus expresiones en el ciberespacio, donde no solo vemos su cotidianidad, sino que es parte de ella en la convocatoria a una manifestación, en las imágenes de una protesta o en los blogs de opinión libre sobre los hechos que les acontecen.

La participación virtual en los movimientos sociales se produce desde el momento en el que las personas se suman a un evento, muestran interés por ser partícipes de una actividad o expresan una opinión sobre la misma, aunque sea mediante el «me gusta» en una red social virtual; expresión que debe ser reconocida como parte de la propia construcción de participación de los/as jóvenes. Al respecto, no podemos dejar de mencionar que, a la vez, se distinguen diversas formas de participación virtual que comprenden desde la información por estos medios, hasta la asociación, la coordinación y la acción desde lo virtual. 
Es en las redes sociales virtuales donde los/as jóvenes han encontrado nuevos espacios de horizontalidad, donde no solo se producen relaciones más cercanas a la individuación sino en los que también se pueden situar sus ideas, sus propuestas y/o sus acciones. De esta manera, se puede reafirmar que «la horizontalidad de las redes favorece la colaboración y la solidaridad, socavando las necesidades de un liderazgo formal» (Castells, 2012, p. 215). Es en los movimientos sociales, entonces, donde se expresa no solo la organización desde lo horizontal, sino también lo paralelo a las organizaciones formales e institucionalizadas. Son estos espacios los que permiten el surgimiento de elementos creativos y autónomos desde donde surgen, muchas veces, ideas y propuestas que se terminan globalizando. Ejemplo de ello es la manera en la que el movimiento estudiantil en Chile realizó la movilización denominada «Genkidama de la Educación», que consistió en una convocatoria virtual, generada dentro del movimiento juvenil pero paralela a la organización institucional, mediante la cual se convocó a cientos de jóvenes a expresarse por las demandas del movimiento estudiantil.

Si bien es cierto que realizaron una coreografía poco entendible para la gran mayoría de los/as adultos/as que observaban en las calles de la ciudad, esta guardaba un gran significante para la población joven, no solamente por el apoyo y por la convocatoria que tuvo dentro del movimiento, sino por lo transversal que resultó desde la organización formal la inserción de elementos propios de socialización cultural. El simbolismo implícito de esta representación está en la forma representativa de la lucha desde la pureza de la base que da sentido al movimiento social, asociado a elementos representativos de la cultura juvenil, donde hay un factor sociocultural característico que es transversal a ellos/as y que guardó un significante por medio de la manifestación de este «Genkidama» por la educación. A lo que se debe agregar que la organización y la convocatoria se realizaron íntegramente desde las redes sociales virtuales, e involucraron la participación de varios actores de diferentes partes del orbe que apoyaron con la acción colectiva.

No es solamente el ejemplo del «Genkidama de la educación», también podemos hacer mención a los diferentes «flashmobs» convocados durante el movimiento, ya sea en base a representaciones musicales -como el «Thriller por la educación», el «Gagazo por la educación», los bailes masivos de axé y hasta una versión orquestada de «El baile de los que sobran»-, todos ellos con acento en los elementos culturales de los/as jóvenes, así como aquellos convocados para representaciones 
puntuales -como la realización de un suicidio simbólico por la educación- o las altas convocatorias a cicletadas y skaters por las avenidas principales. Ligado a aquello se encuentran los Cosplay, en los que se representan peleas entre superhéroes y villanos, o la marcha de los «300 espartanos por la educación».

Entender en esta creatividad a la generación, a partir de sus elementos culturales y estéticos distintivos en las demandas de los/as jóvenes, permite no solo su difusión sino, también, construir respuestas acordes a sus características glocales. Parte del problema estriba en que una buena proporción del análisis tradicional de los movimientos sociales ( $y$, más en general, de la acción colectiva) ha ignorado o ha dejado explícitamente de lado cuestiones de cultura o de interpretación del significado. Esto tiende a apartar la atención de los movimientos ampliamente relacionados con valores, normas, lenguajes, identidades y comprensiones colectivas -incluso las de los/as propios/as participantes en los movimientos- y a dirigirla a los que se centran de modo instrumental en la transformación de las instituciones políticas y económicas (Aguilera, 2014).

Afirmamos, una vez más, que estas características por sí solas no son las que sostienen los movimientos sociales, pero sí desde la generación glocal son facilitadores y aceleradores de su dinámica interna. Lo que hace falta es voltear las miradas para entender a las juventudes, que se encuentran en permanente dinámica, desde sus propios espacios, pero con una mirada propia desde la glocalización que se refleja, fuertemente, en los movimientos glocales de la juventud.

\section{Referencias}

Aguilera, O. (2014). Generaciones: movimientos juveniles, políticas de la identidad y disputas por la visibilidad en el Chile neoliberal. Ciudad Autónoma de Buenos Aires, Argentina: Consejo Latinoamericano de Ciencias Sociales (CLACSO).

Alpízar, L, y Bernal, M. (2003). La construcción social de las juventudes. Última década, 11(19), 105-123. Recuperado de https://ultimadecada.uchile.cl/index.php/UD/article/view/56547

Beck, U. (2008). Generación global. Barcelona, España: Paidós. 
Castells, M. (2012). Redes de indignación y esperanza. Los movimientos sociales en la era de Internet. Madrid, España: Alianza.

Croce, A. (18 de julio de 2012). Nuevas realidades juveniles en América Latina. América Latina en movimiento, (477). Recuperado de https://www.alainet.org/es/active/56602

Cuadra, Á. (9 de agosto de 2012). Cultura de la protesta: protesta de la cultura. América Latina en movimiento, (477). Recuperado de https://www.alainet.org/es/active/57119

Duarte Quapper, K. (2000). ¿Juventud o juventudes? Acerca de cómo mirar y remirar a las juventudes de nuestro continente. Última década, 8(13), 59-77. Recuperado de https://ultimadecada.uchile.cl/index.php/UD/article/view/56498

Feixa, C. (2000). Generación @. La juventud en la era digital. Nómadas, (13), 75-91. Recuperado de https://www.redalyc.org/articulo.oa?id=105115264007

Feixa, C. (2006). Generación XX. Teorías sobre la juventud en la era contemporánea. Revista Latinoamericana de Ciencias Sociales, Niñez y Juventud, 4(2), 21-45. Recuperado de http://www.scielo.org.co/scielo.php?script=sci_arttext\&pid=S1692715X2006000200002

Feixa, C. y Nilan, P. (2009). ¿Una juventud global? Identidades híbridas, mundos plurales. Educación Social. Revista de intervención socioeducativa, (43), 73-87. Recuperado de https://www.raco.cat/index.php/EducacioSocial/article/view/180643

Ferrer, A. (31 de octubre de 2010). Millennials, la generación del siglo XXI. Nueva Revista de Política, Cultura y Arte, (130). Recuperado de https://www.nuevarevista.net/sociedad/millennials-la-generacion-delsiglo-xxi/ 
Lipovetsky, G. [1986] (2000). La era del vacío. Ensayo sobre el individualismo contemporáneo (Trad. Joan Vinyoli y Michèle Pendanx). Barcelona, España: Anagrama.

Maffesoli, M. (1998). El tiempo de las tribus. Barcelona, España: Icaria.

Margulis, M. y Urresti, M. (1998). «La construcción social de la condición de juventud». En H. Cubides, M. C. Laverde y C. E. Valderrama (Eds.), Viviendo a toda: jóvenes, territorios culturales y nuevas sensibilidades (pp. 5-21). Santafé de Bogotá, Colombia: Siglo del Hombre.

Mielli, R. (23 de julio de 2012). Protagonismo juvenil ocupa las calles y las redes. América Latina en Movimiento, (477). Recuperado de https://www.alainet.org/es/active/56715

Piscitelli, A. (16 de agosto de 2005). Nativos digitales vs. Inmigrantes digitales. Recuperado de https://www.filosofitis.com.ar/2005/08/16/nativos-digitales-vsinmigrantes-digitales/

Ramirez, F. (2014). Los nuevos movimientos glocales juveniles. Trabajo presentado en el Primer Seminario Latinoamericano y del Caribe «Escenarios del debate contemporáneo del Trabajo Social y sus proyecciones regionales: tendencias y desafíos». Santiago de Chile, Chile.

Robertson, R. y White, K. E. (2004). «La Glocalizzazione Rivisitata ed Elaborata». En F. Sedda (Ed.), Glocal. Sul presente a venire (pp. 13-41). Roma, Italia: Sossella.

Wallerstein, E. (17 de octubre de 1999). A ruina do capitalismo [entrevista]. Folha de Sao Paulo. Recuperado de https://www1.folha.uol.com.br/fsp/mais/fs1710199912.htm 